\title{
Organized Chronic Subdural Hematoma: A Condition Presenting Mixed-Density Hematoma without Gradation Density on Brain CT
}

\author{
Tetsuya Akaishi $^{a}$ Hiroshi Karibe $^{b}$ Toshiki Endo $^{c}$ Tadashi Ishii $^{a}$ \\ aDepartment of Education and Support for Regional Medicine, Tohoku University \\ Hospital, Sendai, Japan; b Department of Neurosurgery, Sendai City Hospital, Sendai, Japan; \\ 'Department of Neurosurgery, Tohoku University Graduate School of Medicine, Sendai, Japan
}

\section{Keywords}

Chronic subdural hematoma · CT value · Gradation density · Organized subdural hematoma · Urgency

\begin{abstract}
Organized chronic subdural hematoma is a neurological condition characterized by organized clot formation inside the hematoma. Unlike nonorganized chronic subdural hematoma with persistent bleeding inside, organized subdural hematoma is often a nonurgent condition that does not require emergency drainage, unless progressive cerebral compression with neurological disturbances is present. However, as the CT values in organized clot formation and active bleeding fall in similar ranges, distinguishing these 2 conditions with different emergency levels based on brain CT alone is sometimes difficult. Here, we describe the case of a 78-year-old man with persistent nocturia who was referred to our hospital. He had a history of head trauma on the left side about 1 year ago, followed by neurosurgery at another hospital. Brain CT revealed a multiseptated subdural space and a mixed-density hematoma without gradation density on the contralateral side of the past head trauma region. Based on the lack of progressive neurological disturbances and the absence of gradation density in CT values inside the hematomas, his condition was judged as nonurgent, and emergency drainage was spared. One year after his first visit to our hospital, the patient experienced a normal life without any neurological disturbances, except for nocturia. The absence of gradation density in $\mathrm{CT}$ values along the gravity direction inside the organized hematoma may be a clue to estimate nonurgency without persistent active bleeding inside.
\end{abstract}




\section{Introduction}

Organized chronic subdural hematoma is a condition characterized by clot formation and/or calcification that shows multiple lobulations with mixed-density hematoma on brain CT. Unlike the usual nonorganized chronic subdural hematoma, which often requires emergency burr-hole drainage upon progressive neurological symptoms, most patients with organized chronic subdural hematoma do not present progressive conditions and do not require emergency surgery. However, as the organized segment shows elevated CT values on brain CT, discriminating these 2 conditions with different emergency levels is not always easy. In this report, we describe a case of organized chronic subdural hematoma that did not require emergency surgery, for which an absence of gradation in the CT value along the direction of gravity was useful to judge the condition as nonurgent without prolonged active bleeding inside the hematoma.

\section{Case Presentation}

A 78-year-old man was referred by his family doctor to our hospital with prolonged nocturia and no other complaints. His medical history included hypertension, type 2 diabetes mellitus, dyslipidemia, hyperuricemia, and alcoholic liver disease. He had no history of taking antithrombotic medications. About 1 year ago, the patient sustained a fall and severely hit the left side of his head on a door. He visited another general hospital, where he underwent a brain CT without any significant findings, and was regularly followed up with brain CT by the neurosurgeons every 3 months.

At the first visit to our hospital, his neurological examination was normal, including muscle strength in the 4 extremities and orientation level. Brain CT revealed a multiseptated subdural space and a mixed-density hematoma without gradation on the contralateral side of the head injury (Fig. 1a-c). No midline shift was observed. Based on the presence of a highdensity area within the subdural space, persistent bleeding was initially suspected, and emergency consultation with a neurosurgeon was considered. The patient was swiftly consulted with the neurosurgeons of the general hospital who regularly followed the patient. After discussing the estimated urgency of the case carefully, his condition was judged as nonurgent based on the normal neurological findings and the absence of gradation density in the CT values inside the hematoma. The patient was thus diagnosed with an organized chronic subdural hematoma that did not require urgent burr-hole drainage. By retrospectively reviewing the routinely followed previous brain CT images taken at the previous hospitals, the formation of organized areas with high CT values in the hematoma space was suggested to have started after 3-6 months from the first head trauma episode. One year after his first visit to our hospital, surgery was not required, and the patient experienced a normal life without any neurological disturbances, except for the nocturia.

\section{Discussion}

The ideal management for patients with organized subdural hematoma remains controversial, and surgical procedures may be spared in some cases [1,2]. Because the CT values of the organized segments are usually not as high as those for calcification (which usually presents as values $>100 \mathrm{HU}$ ), it is difficult to distinguish the two conditions from brain CT images alone [3]. Certainly, the organized areas in the present case showed CT values of 40-80 HU, which fit the range of CT values for hematoma with active bleeding inside. One

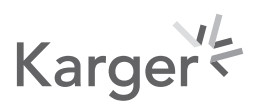




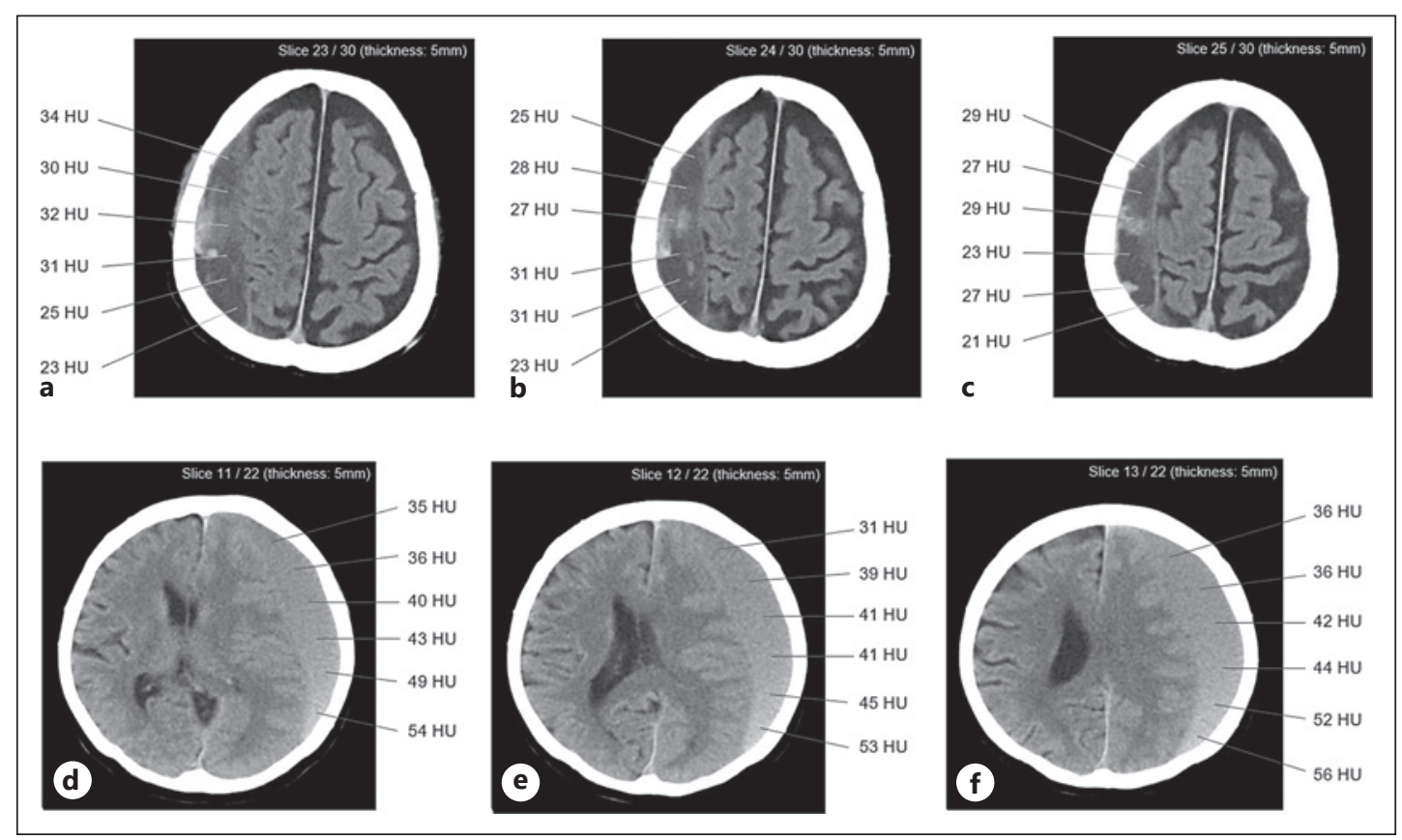

Fig. 1. Acute CT images of a nonurgent case with organized cerebral subdural hematoma (panels a-c) revealed multiseptated mixed-density hematoma without gradation inside. Meanwhile, CT images of another urgent case (panels $\mathbf{d}-\mathbf{f}$ ) that required emergency burr-hole drainage showed gradation density hematoma. The values beside each CT image are those measured at the point indicated within the hematoma.

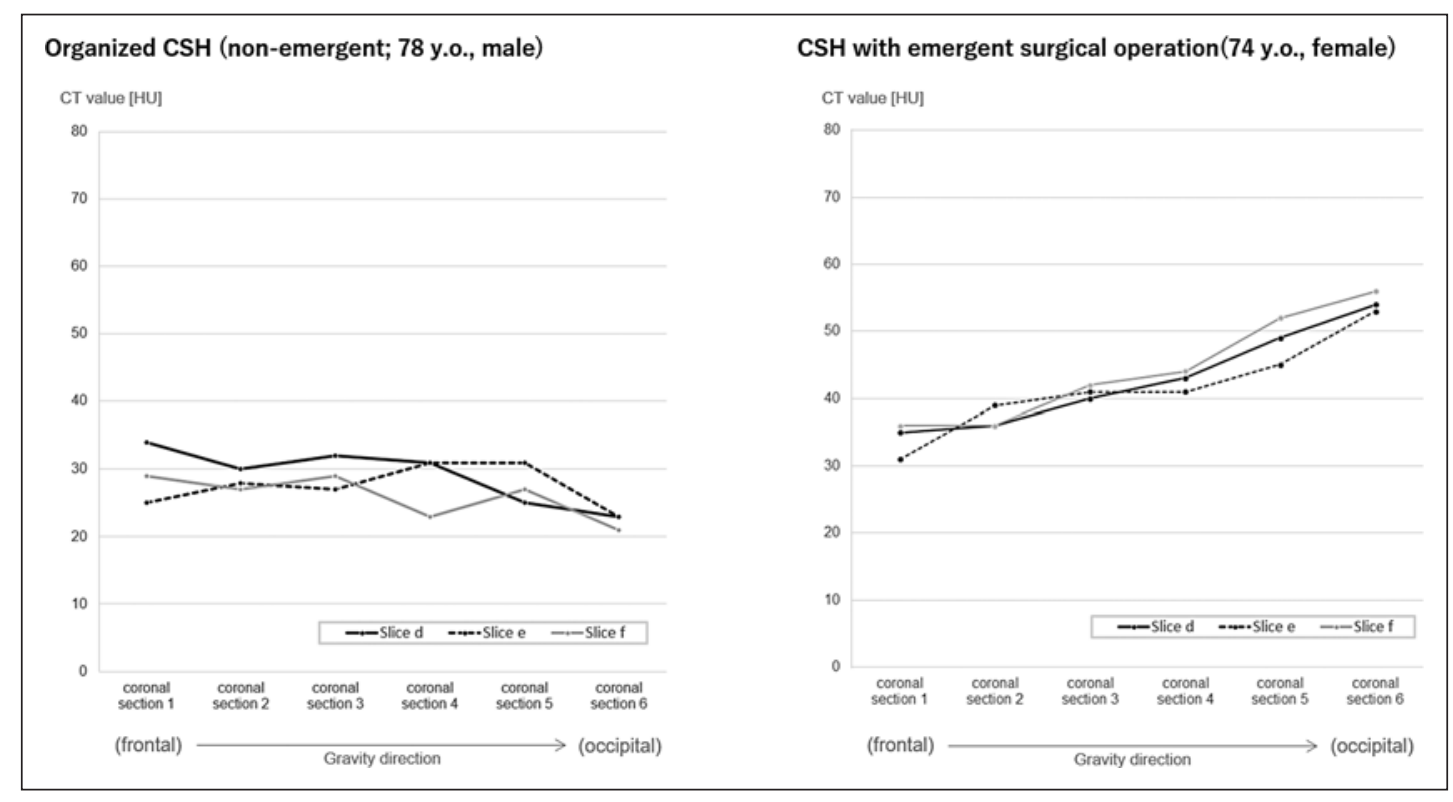

Fig. 2. Line graphs of the measured CT values along the gravity direction (i.e., fronto-occipital direction) from 6 equally spaced regions on 3 different consecutive axial slices from the 2 patients are shown. Intra-hematoma smooth gradation density in CT values was absent in the nonurgent case with organized hematoma (left), whereas it was readily observed in another urgent case with nonorganized chronic subdural hematoma (right). Slices a-f correspond to the slice levels of panels a-f in Figure 1. 
of the possible clues suggesting urgency is the presence of a gradation density inside the hematoma on brain CT, suggestive of persistent bleeding [4]. Three slices of brain CT images with gradation from another 74-year-old female subject with chronic subdural hematoma that required emergency burr-hole drainage are shown in Figure $1 \mathrm{~d}-\mathrm{f}$. A gradation density hematoma with a smooth color change along the gravity direction was apparent, suggesting the presence of persistent, acute bleeding in the hematoma. Line graphs of the measured CT values from 6 equally spaced regions (excluding the organized areas) along the gravity direction in each of the 2 patients are shown in Figure 2. The presence of grade density in the CT values was present only in the urgent case who required emergency surgery, and it was not seen in the nonurgent case with organized chronic subdural hematoma. Lastly, although the intra-hematoma gradation density in CT values would be useful to judge the urgency, it should be noted that the presence of cerebral compression with progressive neurological symptoms and rapid change in hematoma volume are the current standards for judging the urgency in each patient [5]. Clinicians should carefully decide the urgency in each case with chronic subdural hematoma based on multiple factors, including the presence of gradation density in CT values that implies persistent active bleeding inside the hematoma.

\section{Statement of Ethics}

Written informed consent was obtained from the 2 patients for publication of their clinical history and images. The author's institutions do not require ethical approval for the publication of a single case or case series.

\section{Conflict of Interest Statement}

The authors have no conflicts of interest to disclose.

\section{Funding Sources}

The authors received no financial support for the present report.

\section{Author Contributions}

Tetsuya Akaishi contributed to conception, evaluation, investigation, diagnosis, and management of the patient and drafted the manuscript. Hiroshi Karibe contributed to evaluation, investigation, diagnosis, management, and follow-up of the patient and critically revised the manuscript. Toshiki Endo contributed to evaluation, diagnosis, and management of the patient, supervision, and critical revision of the manuscript. Tadashi Ishii contributed to evaluation and investigation of the patient, supervision, and critical revision of the manuscript.

\section{Data Availability Statement}

Details supporting the findings of this study are available from the corresponding author on reasonable requests from qualified clinicians or researchers. 


\section{References}

1 Pappamikail L, Rato R, Novais G, Bernardo E. Chronic calcified subdural hematoma: case report and review of the literature. Surg Neurol Int. 2013;4:21.

2 Yokoya S, Nishii S, Takezawa H, Katsumori T, Takagi Y, Goto Y, et al. Organized chronic subdural hematoma treated with middle meningeal artery embolization and small craniotomy: two case reports. Asian J Neurosurg. 2020 Apr-Jun;15(2):421-4.

3 Nute JL, Le Roux L, Chandler AG, Baladandayuthapani V, Schellingerhout D, Cody DD. Differentiation of lowattenuation intracranial hemorrhage and calcification using dual-energy computed tomography in a phantom system. Invest Radiol. 2015 Jan;50(1):9-16.

4 Shimizu Y, Park C, Tokuda K. Gradation density hematoma is a predictor of chronic subdural hematoma recurrence associated with inflammation of the outer membrane. Clin Neurol Neurosurg. 2020 Jul; 194:105839.

5 Imaizumi S, Onuma T, Kameyama M, Naganuma H. Organized chronic subdural hematoma requiring craniotomy: five case reports. Neurol Med Chir. 2001 Jan;41(1):19-24. 